\title{
Association between low eosinophil count and mortality in SARS-COV-2 infection on the 70+, a discussed observation
}

Edouard Baudouin ( $\nabla$ edouardpierre.baudouin@aphp.fr)

Service Hospitalo-Universitaire de gériatrie. Assistance Publique-Hôpitaux de Paris, Hôpitaux Universitaires Paris- Saclay, Hôpital Paul-Brousse- Villejuif

Jill Kosowki

Service Hospitalo-Universitaire de gériatrie. Assistance Publique-Hôpitaux de Paris, Hôpitaux Universitaires Paris-Saclay, Hôpital Paul-Brousse- Villejuif

\section{Lea Mesinele}

Service Hospitalo-Universitaire de gériatrie. Assistance Publique-Hôpitaux de Paris, Hôpitaux Universitaires Paris-Saclay, Hôpital Paul-Brousse- Villejuif

\section{Tom Pujol}

Service Hospitalo-Universitaire de gériatrie. Assistance Publique-Hôpitaux de Paris, Hôpitaux Universitaires Paris-Saclay, Hôpital Paul-Brousse- Villejuif

\section{Nicoletta Brunetti}

Service Hospitalo-Universitaire de gériatrie. Assistance Publique-Hôpitaux de Paris, Hôpitaux Universitaires Paris-Saclay, Hôpital Paul-Brousse- Villejuif

\section{Marion Colas}

Service Hospitalo-Universitaire de gériatrie. Assistance Publique-Hôpitaux de Paris, Hôpitaux Universitaires Paris-Saclay, Hôpital Paul-Brousse- Villejuif

\section{Marie Neiss}

Service Hospitalo-Universitaire de gériatrie. Assistance Publique-Hôpitaux de Paris, Hôpitaux Universitaires Paris-Saclay, Hôpital Paul-Brousse- Villejuif

\section{Pauline Simon}

Service Hospitalo-Universitaire de gériatrie. Assistance Publique-Hôpitaux de Paris, Hôpitaux Universitaires Paris-Saclay, Hôpital Paul-Brousse- Villejuif

\section{Christophe Trivalle}

Service Hospitalo-Universitaire de gériatrie. Assistance Publique-Hôpitaux de Paris, Hôpitaux Universitaires Paris-Saclay, Hôpital Paul-Brousse- Villejuif

\section{Soraya Tiramine}

Service Hospitalo-Universitaire de gériatrie. Assistance Publique-Hôpitaux de Paris, Hôpitaux Universitaires Paris-Saclay, Hôpital Paul-Brousse- Villejuif

\section{Fahd Laraaj}


Service Hospitalo-Universitaire de gériatrie. Assistance Publique-Hôpitaux de Paris, Hôpitaux Universitaires Paris- Saclay, Hôpital Paul-Brousse- Villejuif

\section{Anne-Laure Vetillard}

Service Hospitalo-Universitaire de gériatrie. Assistance Publique-Hôpitaux de Paris, Hôpitaux Universitaires Paris- Saclay, Hôpital Paul-Brousse- Villejuif

\section{Nadège Houenou-Quenum}

Service Hospitalo-Universitaire de gériatrie. Assistance Publique-Hôpitaux de Paris, Hôpitaux Universitaires Paris- Saclay, Hôpital Paul-Brousse- Villejuif

\section{Cecile Souques}

Service Hospitalo-Universitaire de gériatrie. Assistance Publique-Hôpitaux de Paris, Hôpitaux Universitaires Paris- Saclay, Hôpital Paul-Brousse- Villejuif

\section{Sébastien Verdier}

Service Hospitalo-Universitaire de gériatrie. Assistance Publique-Hôpitaux de Paris, Hôpitaux Universitaires Paris- Saclay, Hôpital Paul-Brousse- Villejuif

\section{Julie Houdre}

Service Hospitalo-Universitaire de gériatrie. Assistance Publique-Hôpitaux de Paris, Hôpitaux Universitaires Paris- Saclay, Hôpital Paul-Brousse- Villejuif

\section{Adrien Sorrel-Dejerine}

Service Hospitalo-Universitaire de gériatrie. Assistance Publique-Hôpitaux de Paris, Hôpitaux Universitaires Paris- Saclay, Hôpital Paul-Brousse- Villejuif

\section{Joge Sanchez}

Service Hospitalo-Universitaire de gériatrie. Assistance Publique-Hôpitaux de Paris, Hôpitaux Universitaires Paris- Saclay, Hôpital Paul-Brousse- Villejuif

\section{Vidal Jean-Sebastien}

Hôpital Broca

\section{Magali Guichardon}

Service Hospitalo-Universitaire de gériatrie. Assistance Publique-Hôpitaux de Paris, Hôpitaux Universitaires Paris- Saclay, Hôpital Paul-Brousse- Villejuif

\section{Kahn Jean-Emmanuel}

Service Hospitalo-Universitaire de gériatrie. Assistance Publique-Hôpitaux de Paris, Hôpitaux Universitaires Paris- Saclay, Hôpital Ambroise Paré- Bologne-Billancourt

\section{Rocca Collarino}

Service Hospitalo-Universitaire de gériatrie. Assistance Publique-Hôpitaux de Paris, Hôpitaux Universitaires Paris- Saclay, Hôpital Paul-Brousse- Villejuif

\section{Emmanuelle Duron}

Service Hospitalo-Universitaire de gériatrie. Assistance Publique-Hôpitaux de Paris, Hôpitaux Universitaires Paris- Saclay, Hôpital Paul-Brousse- Villejuif

\section{Research Article}


Keywords: SARS-COV-2, geriatrics, in-hospital mortality, low eosinophil count

Posted Date: October 20th, 2021

DOI: https://doi.org/10.21203/rs.3.rs-378188/v2

License: (c) (1) This work is licensed under a Creative Commons Attribution 4.0 International License. Read Full License 


\section{Abstract}

Background: Biological COVID-19 abnormalities are varied (lymphopenia, high levels of blood inflammatory markers, ...). Regarding physiological immune responses, eosinophils were for a long time thought to be mainly against extra-cellular pathogen like helminthes. It has recently been pointed out that they appear to play a part in recognition of virus and to have the capacity to perform direct action against viruses. This study aimed to seek for an association between eosinophils count $<10 / \mathrm{mm} 3$ and mortality in an older adults suffering from COVID-19 hospitalized in a specific geriatric ward.

Methods: This observational retrospective study was conducted in a French geriatric ward from March 17 to April 18, 2020. All 118 patients hospitalized for COVID-19 over 70 yo in acute stay care were enrolled. Patients with a treatment or a condition which could interfere with eosinophil count were excluded.

Results: No statistical difference was found between surviving or deceased patient regarding age (mean age (SD): 87 years (7)) and sex (34\% of males). Differences for the most frequent acute events were statically different: Quick Sepsis-related Organ Failure Assessment (qSOFA) score was $\geq 2$ at admission for $23 \%$ in the survivor group vs $72 \%$ in the deceased $(p<.001)$; acute kidney injury concerned $17 \%$ of the survivors vs. $69 \%$ of the decease $(p<.001)$. Eosinophil count $<10 / \mathrm{mm} 3$ was significantly associated with mortality $(\mathrm{OR}(95 \% \mathrm{Cl}))=3.54(1.23-11.4)$ after adjustment on age, gender, and activity of daily living.

Conclusion: Low eosinophil count $(<10 / \mathrm{mm} 3)$ seems to be associated with mortality on older adults. Due to the specific physiological ageing process of the immune system, this calls to explore more globally the impact of inflammaging and immunosenescence on SARS-CoV-2 infection in this population.

\section{Introduction}

SARS-COV-2 outbreak, stated in winter 2020 as a public health emergency of international concerned by the World Health Organization (WHO) disrupted societies, health systems and scientific paradigms: everything needed to be learned. Indeed, due to its large spreading and lethality, the understanding of its physiopathology as much as finding prognostic factors are needed for health system to be re-organized and clinician to make decisions. It has affected every country, resulting in more than 238 million identified cases with 4 million confirmed deaths since December 2019 (1). In this context, our elders (a world growing population) seem more vulnerable: since the first of March, $73 \%$ of the deceased were 75 years old or more in France (2).

Age has indeed been described to be a major independent prognostic factor, as hypertension, diabetes and obesity (3-7). Of note, our deceased in a geriatric COVID-19 ward this fall were highly disabled as shown by Zerah and al (8). Their activity of daily living (ADL) - scored over 6 which marks no disability (9) - was significantly associated with mortality: $A D L \leq 3$ had an OR of $1.84(1.25-2.70)$ on the multivariate analysis. However, the age adjusted Charlson comorbidity index (10) did not seem to be a significant prognostic variable on mortality status: median (IQR) were $7(6-9)$ vs $7(6-8) p=0.28$. 
Biological COVID-19 abnormalities include lymphopenia, increased levels of blood inflammatory markers (such as C-reactive protein (CRP), ferritin, ...) and a high lactate dehydrogenase (LDH) blood

level (11). Regarding physiological aspect, immune responses are usually classified in three types against pathogens: type 1 against intra-cellular pathogen including viruses, type 2 against extra-cellular pathogen like helminthes and type 3 against extra-cellular pathogen like bacteria and fungus (12). Eosinophils were for a long time included in the type 2 response and explored for their implication in allergic or pulmonary diseases (13). Their role appears to be wider as they have been linked to immune response conferring host protection against fungi, bacteria, and viruses. They seem to be able to play a part in recognition of virus and to have the capacity to perform direct action against viruses (13).

However, literature between eosinophils and infectious diseases - helminthes apart - appears to be scarce. One study found that eosinophil count under $40 / \mathrm{mm} 3$ with elevated white cell count (over $10,000 / \mathrm{mm} 3$ ) was associated with bacterial infectious diseases $(14,15)$. Another study found that eosinophils $<10 / \mathrm{mm}^{3}$ alone or in association with elevated white blood cell count or high CRP blood level was strongly associated with sepsis (specificity 94-98\%) in an emergency department (16). A third study found that eosinophils $<40 / \mathrm{mm} 3$ was a strong (all-cause) mortality predictor (Hazard Ratio 1.85 $(95 \% \mathrm{Cl} 1.01-3.42), p=0.046)$ in intensive care unit (17). Along with these observations, studies on SARSCOV-2 found that low count seemed to be correlated with the prognosis (18-21). Interestingly, eosinophil levels increased in patients prior discharge, suggesting that following their rate might help to monitor betterment (22). Although it remains unclear, low eosinophil count for some authors involves inhibition of eosinophil life cycle, apoptosis may be induced by interferon type 1 during acute infection and eosinophil consumption through eosinophil antiviral actions(13).

In this way, low eosinophil count could be a prognosis marker for mortality $(17,23,24)$; however, none of these reports included geriatric patients (over $75 \mathrm{y} .0$ with significant comorbidities and disability).

Thus, this study analyzed the association between low eosinophil count $(<10 / \mathrm{mm} 3)$ and mortality in older adults hospitalized in a specific geriatric COVID-19 ward.

\section{Materials And Methods}

\section{Study Design, Setting, and Participants}

This monocentric retrospective cohort was an ancillary study of the Zerah and al cohort which took place from March 13 to April 152020 (8). Informed consent was obtained for all participants and this study was approved by the University Hospital of Paris research committee on April 17, 2020 and by the institutional ethics board of Sorbonne University on May 11, 2020 (2020-CER-2020-43). This report follows the STROBE recommendations.

Inclusion criteria were: at least age 70 years old and hospitalization in a geriatric ward for COVID-19. Noninclusion criteria were conditions or medications that might modify eosinophil count: 
immunosuppression (HIV with CD4 < 200/ mm3), chronic corticosteroid use, asthma, chemotherapy or immunosuppressive agents, documented lymphoproliferative or myeloproliferative disorders and documented parasitic diseases.

The diagnosis of COVID-19 was confirmed by a nasopharyngeal reverse transcription polymerase chain reaction (RT-PCR) for SARS-CoV-2, according to the WHO guidance (25).

\section{Data Collection Methods and Data Management}

Patients' medical records were reviewed and analyzed by trained physicians. We included baseline characteristics before COVID-19-19: age, gender, community or nursing home residence, previous medical history, and chronic medications. Recorded comorbidities were: atrial fibrillation, active cancer, dementia, depression, diabetes, congestive heart failure, coronary artery disease, thromboembolic disease, hemiplegia, stroke, Chronic Obstructive Pulmonary Syndrom (COPD), peripheral arterial obstructive disease, hypertension, Parkinson disease chronical kidney disease. Severity was assessed with the Charlson's index (10) and functional status was assessed by the Activities of Daily Living (ADL) scale (6 basic human functions: bathing, dressing, toileting, transfer, continence, and feeding; 1 point for each function (9)). ADL was categorized in a binary variable: $A D L<3$. According to French health authority (Haute Autorité de Santé), eosinophils count respectively for male and female over 65 y.o is $50 / \mathrm{mm} 3$ $690 / \mathrm{mm} 3 ; 40 / \mathrm{mm} 3-450 / \mathrm{mm} 3$ (2.5 percentile -97.5 percentile)(26). Due to the very low eosinophils count in this cohort, cut-off for low eosinophil count was set to $<10 / \mathrm{mm} 3$ because it has been associated with poor outcome during sepsis and defined absolute eosinopenia (16).

Acute events were recorded: atrial fibrillation, heart failure, pulmonary edema, disorders of consciousness defined by Glasgow score < 14, thromboembolic or hemorrhagic event, acute kidney failure identified according to the Kidney Disease Improving Global Outcomes (KDIGO) definition (27), fecal impaction, acute urinary retention, pressure ulcer.

Quick Sepsis-related Organ Failure Assessment $\geq 2$ (qSOFA), defined by a range 0-3, with 1 point each for systolic hypotension ( $\leq 100 \mathrm{mmHg}$ ), tachypnea ( $\geq 22 / \mathrm{min}$ ) or altered consciousness (Glasgow coma score $<14$ ) was recorder (28). Biological data during infection were also collected: white blood cell count, neutrophil count, eosinophil count, lymphocytes count, platelets count, hemoglobin level, presence of liver injury (aspartate aminotransferase or alanine aminotransferase $\geq 2$ times normal level) and cholestasis with alkaline phosphatase or gamma-glutamyltransferase $\geq 2$ times the upper limit of normal. Data at discharge were also reported: vital status (alive or dead), length of stay and discharge location (home; nursing home; long term rehabilitation center or other).

\section{Statistical analysis}


As we included all patients from the center, no a priori power calculation was done. Normality of variable was assessed by a graphical representation of their distribution. Data are presented with mean and standard deviation (SD) for continuous variables and count (percentage) for categorical variables. T-tests were used for continuous variables and chi-squared tests or Fisher's exact tests for categorical variables. Patient characteristics are described overall and according to mortality status during the stay in the acute geriatric stay.

Missing values represented overall less than $8 \%$ of all of the data and $4 \%$ for the variable used in the multivariate analysis. Thus, no imputation of the missing data was performed.

We performed exploratory analysis in order to assess for other associated factors with mortality and their correlation by a focused principal components analysis.

We performed a logistic regression with adjustment for age, sex and $A D L>3$. ADL was chosen because it was a significant factor in the main study and is a relevant geriatric variable. No stepwise was performed due to the limited power and the number of missing data.

Analyses were performed with R V4.0.0.

\section{Results}

One hundred and eighteen patients were included during the study period. The mean (SD) length of stay was 12.3 (7.4) days with a statistical difference between survivors (13.5 (7.0) days) and deceased (9 (7.5) days). 32 (27\%) patients have died during their stay in our acute COVID-19 ward. As for the survivors, $42(35.6 \%)$ patients were transferred to a rehabilitation ward, $33(28.0 \%)$ patients returned home and $5(4.2 \%)$ were transferred to another ward such as palliative care.

Demographic and clinical data of the population are presented in table 1. No statistical difference was found between surviving or deceased patient regarding age and sex (mean age (SD) was 87 years (7); $p=$ $0.54)$ ) and $40(34 \%)$ were male $(\mathrm{p}=0.07)$. The age adjusted Charlson comorbidity index was high in both groups with a trend for a higher score for the deceased (mean (SD) $=4.7(2.7)$ vs $5.7(2.9), p=0.10$ ), as well as a more frequent $A D L$ score $<3(47(55 \%)$ for survivors vs. $23(72 \%), p=0.06)$.

Table 1: Baseline characteristics and acute events 


\begin{tabular}{|c|c|c|c|c|}
\hline \multirow[t]{2}{*}{ Characteristics } & Total & Survivor & Deceased & \multirow[t]{2}{*}{$p$} \\
\hline & $\mathrm{n}=118$ & $n=86(73 \%)$ & $\mathrm{n}=32(27 \%)$ & \\
\hline \multicolumn{5}{|l|}{ Socio-demographic data } \\
\hline Age, mean $(S D)^{\star}$ & $87.0(7)$ & $86.7(6.2)$ & $87.6(6.7)$ & 0.54 \\
\hline Male, $n(\%)$ & $40(34.0)$ & $25(29.1)$ & $15(47.0)$ & 0.07 \\
\hline \multicolumn{5}{|l|}{ Comorbidities, mean (SD) } \\
\hline Adjusted Charlson comorbidity Index & $5.0(2.8)$ & $4.7(2.7)$ & $5.7(2.9)$ & 0.10 \\
\hline Number of treatments & $7.3(3.5)$ & $7.3(3.6)$ & $7.3(3.2)$ & 0.98 \\
\hline \multicolumn{5}{|l|}{ Comorbidities, n (\%) } \\
\hline Atrial fibrillation & $43(36.4)$ & $27(31.4)$ & $16(50.0)$ & 0.06 \\
\hline Active cancer & $14(11.9)$ & $11(12.8)$ & $3(9.4)$ & 0.76 \\
\hline Dementia & $97(82.2)$ & $69(80.2)$ & $28(87.5)$ & 0.36 \\
\hline Depression & $66(55.9)$ & $51(59.3)$ & $15(46.9)$ & 0.23 \\
\hline Diabetes & $35(29.7)$ & $24(27.9)$ & $11(34.4)$ & 0.49 \\
\hline Congestive heart failure & $39(33.1)$ & $25(29.1)$ & $14(43.8)$ & 0.13 \\
\hline Coronary artery disease & $32(27.1)$ & $21(24.4)$ & $11(34.4)$ & 0.28 \\
\hline Thromboembolic disease & $17(14.4)$ & $13(15.1)$ & $4(12.5)$ & 0.99 \\
\hline Hemiplegia & $10(8.5)$ & $6(7.0)$ & $4(12.5)$ & 0.46 \\
\hline Stroke & $32(27.1)$ & $16(18.6)$ & $16(50)$ & $<.001$ \\
\hline COPD & $14(11.9)$ & $10(11.6)$ & $4(12.5)$ & 0.99 \\
\hline Peripheral arterial obstructive disease & $14(11.9)$ & $7(8.1)$ & $7(22)$ & 0.05 \\
\hline Hypertension & $85(72)$ & $61(71)$ & $24(75)$ & 0.66 \\
\hline Parkinson disease & $7(5.9)$ & $7(8.1)$ & $0(0)$ & 0.19 \\
\hline Chronical kidney disease & $32(27.1)$ & $19(22.1)$ & $13(40.6)$ & 0.02 \\
\hline \multicolumn{5}{|l|}{ Autonomy } \\
\hline Living in nursing home, $\mathrm{n}(\%)$ & 73 (61.9) & $50(58.1)$ & $23(71.9)$ & 0.25 \\
\hline$A D L<3, n(\%)$ & $70(59.3)$ & $47(54.7)$ & $23(71.9)$ & 0.06 \\
\hline BMI, mean (SD) & $23.1(4.9)$ & $22.9(4.7)$ & $23.8(5.8)$ & 0.52 \\
\hline
\end{tabular}




\begin{tabular}{|lllll|}
\hline Characteristics & Total & Survivor & Deceased & \multirow{2}{*}{$\boldsymbol{p}$} \\
\cline { 2 - 4 } & $\mathrm{n}=118$ & $\mathrm{n}=86(73 \%)$ & $\mathrm{n}=32(27 \%)$ & \\
\hline Acute atrial fibrillation & $4(3.4)$ & $4(4.7)$ & $0(0)$ & 0.57 \\
\hline Acute heart failure & $6(5.1)$ & $3(3.5)$ & $3(9.4)$ & 0.19 \\
\hline Acute coronary event & $1(0.8)$ & $1(1.2)$ & $0(0)$ & 0.99 \\
\hline Glasgow score < 15 & $39(33.1)$ & $12(14.0)$ & $27(84.4)$ & $<.001$ \\
\hline Thromboembolic event & $1(0.8)$ & $1(1.2)$ & $0(0)$ & 0.99 \\
\hline Hemorrhagic event & $5(4.2)$ & $4(4.7)$ & $1(3.1)$ & 0.99 \\
\hline Acute kidney injury & $37(31.4)$ & $15(17.4)$ & $22(68.8)$ & $<.001$ \\
\hline qSOFA $\geq 2$ & $44(37.3)$ & $20(24.4)$ & $23(71.9)$ & $<.001$ \\
\hline Oxygen $\mathrm{n}$ (\%) & & & & $<.001$ \\
\hline None & $54(45.8)$ & $48(55.8)$ & $6(18.8)$ & \\
\hline$<9$ I/min & $52(44.1)$ & $37(43.0)$ & $15(46.9)$ & \\
\hline 9-15 I/min & $12(10.2)$ & $1(1.2)$ & $11(34.4)$ & \\
\hline Specific treatments & & & & \\
\hline Antibiotics introduction, $\mathrm{n}$ (\%) & $75(63.6)$ & $52(60.5)$ & $23(71.9)$ & 0.25 \\
\hline
\end{tabular}

*SD : Standard deviation

Abbreviations: ADL: Activities of Daily Living ; BMI: Body Mass Index; qSOFA: quick Sequential Organ Failure Assessment, Acute kidney injury: identified according to the Kidney Disease Improving Global Outcomes (KDIGO) definition.

QSofa score $\geq 2$ at admission and acute kidney injury were significantly more frequent in the deceased group that in the survivor group (23 (72\%) vs. $20(23 \%)), p<.001)$ and $22(69 \%)$ vs. $15(17 \%), p<.001$ respectively)

Biological data are described in table 2. Regarding blood cells count, eosinophil count $<10 / \mathrm{mm} 3$, was more frequent in the deceased group than in the survivor group (36 patients (42\%) vs. 17 patients (53\%); $p=0.008)$.

Table 2: Laboratory findings 


\begin{tabular}{|c|c|c|c|c|}
\hline \multirow{2}{*}{$\begin{array}{l}\text { Biological characteristics } \\
(\text { mean(SD)) }\end{array}$} & Total & Survivor & Deceased & \multirow[t]{2}{*}{$p$} \\
\hline & $\mathrm{n}=118$ & $\mathrm{n}=86(73 \%)$ & $\mathrm{n}=32(27 \%)$ & \\
\hline Max Hemoglobin (g/dL) & $13.2(1.80)$ & $13.2(1.80)$ & $13.4(2.00)$ & 0.67 \\
\hline Min Hemoglobin (g/dL) & $11.4(1.70)$ & $11.3(1.70)$ & $11.6(1.70)$ & 0.53 \\
\hline Max leukocyte count (mm3) & $7943(2979)$ & 7410 (2699) & 9830 (3209) & $\begin{array}{l}< \\
0.05\end{array}$ \\
\hline Min leukocyte count (/mm3) & $4704(1925)$ & $4320(1172)$ & $6063(3147)$ & 0.01 \\
\hline Max neutrophil count (/mm3) & $5629(2688)$ & 5025 (2249) & 7934 (3019) & $\begin{array}{l}< \\
.001\end{array}$ \\
\hline Min neutrophil count (/mm3) & $3043(1790)$ & $2646(1066)$ & 4495 (2887) & $\begin{array}{l}<.05 \\
0.05\end{array}$ \\
\hline $\begin{array}{l}\text { Max eosinophil count } \\
(/ \mathrm{mm} 3)\end{array}$ & $150(152)$ & $169(160)$ & $79(86)$ & $<.001$ \\
\hline Min eosinophil count (/mm3) & $31(58)$ & $36(62)$ & $13(36)$ & 0.03 \\
\hline Max lymphocyte (/mm3) & $1496(689)$ & $1521(655)$ & $1400(816)$ & 0.53 \\
\hline $\begin{array}{l}\text { Min lymphocyte count } \\
(/ \mathrm{mm} 3)\end{array}$ & $894(512)$ & $920(494)$ & $803(570)$ & 0.37 \\
\hline Max platelet count (/mm3) & $\begin{array}{l}313898(108 \\
902)\end{array}$ & $\begin{array}{l}327529(108 \\
814)\end{array}$ & $\begin{array}{l}263522(95 \\
400)\end{array}$ & 0.01 \\
\hline Min platelet count $(/ \mathrm{mm} 3)$ & 194000 (62 931) & $198518(58$ 771) & $\begin{array}{l}178000(75 \\
095)\end{array}$ & 0.23 \\
\hline Max CRP (/mm3) & $110.8(87.0)$ & $205.1(85.5)$ & $84.2(66.5)$ & $<.001$ \\
\hline Liver Cytolysis, n (\%) & $9(7.60)$ & $4(4.70)$ & $5(15.6)$ & 0.04 \\
\hline Cholestasis, n (\%) & $11(9.30)$ & $5(5.80)$ & 6 (18.8) & 0.02 \\
\hline
\end{tabular}

The multivariate analysis shows that low eosinophil count was associated with an OR of 3.54 (1.23-11.4) after adjustment for age, sex and $A D L<3$ (figure 1).

Figure 1: Multivariate analysis (deceased status adjusted on age, sex, eosinophil count and functional status), Forest Plot

OR (95\% Cl): Age: 1.0 (0.92-1.10), Sex (ref = F): 2.50 (0.80-7.92), Eosinophil count: 3.54 (1.23-11.4), ADL < $3(\mathrm{ref}=\mathrm{No}): 0.36(0.09-1.17)$ 
This study tends to show that eosinophils count< 10/mm3 was associated with mortality: OR (95\% Cl) 3.54 (1.23-11.4) after adjustment for age, gender, and disability (i.e., ADL < 3) older patients suffering from COVID-19. At first glance, these results seem to be in line with literature (29-33) where low eosinophil count is associated with poorer prognostic and conversely, a better prognosis when subjects present eosinophilia due to asthma $(34,35)$.

However, this result does not seem consistent with Lucas and al (12). In this longitudinal analysis of immunological events in moderate and severe COVID-19 on 135 patients, compared to 108 healthy volunteers, they found that severe cases were associated with a higher eosinophil rate compare to moderate and control cases: $p=0.016$ for severe cases vs moderate cases and $p<0.01$ for severe cases vs. controls. In order to discuss this difference between our results and this immunology mapping, some points need to be clarified.

First, in the Lucas and al. cohort, participants were much younger (mean age (SD) = $63(17)$ ) than our participants (mean age (SD) of 87 (7) years old (6)). Immunosenescence and inflammaging, a chronic inflammatory state observed in the elderly where there is an elevation of pro-inflammatory mediators such as including interleukin-1, beta interleukin-6 and tumor necrosis factor alpha (36) might be involved in the differences observed. This chronic pathological state may be worsened during the "cytokine storm" in which it has been found a significant elevation of the same factors on younger subjects and results in an immunodeficiency (37). However, even though inflammation remains the main hypothesis, it is interesting to notice that unlike other cohort (38) in which the cardiovascular burden (hypertension, congestive cardiac heart failure, atrial fibrillation) has been highlighted to be a major prognostic factor were not statistically associated with mortality. This may be explained by the much higher prevalence of these conditions in our study.

Secondly, as exposed in the introduction, eosinophils are likely to be involved during immune responses against viruses. However, it has been described that during an acute inflammation state caused by bacterial infection, there is a drop of circulating eosinophils due to accumulation of eosinophils at the periphery of the inflammatory site, and an inhibition of egress of eosinophils from the bone marrow which leads to inhibition of eosinopoiesis(39)). Furthermore geriatric patients may be more vulnerable to bacterial secondary infection such as aspiration pneumonia due to deglutition disorder, are highly prevalent in geriatric population (44\% in geriatric acute care) (40); the observed low eosinophil count in this study might therefore be in part explained by bacterial secondary infections. Moreover, even though recruitment in lungs tissue does not seem to appear during a COVID-19 infection (41), it has been suggested by Lindsley and al (42) that these low eosinophil count could be the result of a similar mechanism than the one described during bacterial infection. Thus, low eosinophil count might be a sign of host exhaustion imputable to the elimination of COVID-19 virus (43).

Finally, we can notice the significant proportion of Chronical kidney disease and AKI in the deceased group: $40.6 \%$ vs $22.1 \%$; $p=0.027$ and $69 \%$ vs $17 \%, p<.001$ respectively. This point is relevant because as we can find significant link between eosinophilia (44) and kidney diseases, it seems that low eosinophil 
count during SARS-CoV-2 infection might overtake the mechanism that leads to eosinophilia when suffering from kidney injury.

To conclude, one could make the assumption that a poor prognostic of a SARS-CoV-2 infection can, among other factors, be the result of a lack of the antiviral activity and immunomodulation provided by the eosinophils. Hence, it might be a marker to start specific therapy such as antiviral or immunomodulator.

We recognize that the non-inclusion of neutrophils in the multivariate analysis can be a major limit. However, due the conception of the main study, the contemporaneous rate of neutrophils was not accounted for when the minimum of eosinophil count was recorded. Thus, neutrophils' maximum and minimum were not useable in the same model as the lowest eosinophil count.

We acknowledge that the retrospective and monocentric nature of this study limit the scope of the study and preclude any causal inference of the results.

Finally, the small sample size induced an important lack of power, which only enable a limited multivariate analysis.

\section{Conclusions And Implications}

Although this study suffers some limitations, this result if confirmed in other study, gives an additional element for clinician to predict the outcome of a SARS-COV-2 infection on geriatric patients. Indeed, eosinophils count $<10 / \mathrm{mm}^{3}$ tends to be associated with mortality on older adults. Due to the specific physiological ageing process of the immune system, this calls explore more globally the impact of inflammaging and immunosenescence on SARS-CoV-2 infection in this population.

\section{Declarations}

Ethics approval and consent to participate: This study approved by the research committee of the University Hospital of Paris on April 17, 2020 and by the institutional ethics board of Sorbonne University on May 11th, 2020 (2020-CER-2020-43). Informed consent was obtained for all participants. This study is in line with article L1121-1 of French Public Health and French data protection authority.

Consent for publication: Not applicable.

Competing interests: : None of the authors have conflicts of interest to disclose and meet the criteria for authorship as stated in the Uniform Requirements for Manuscripts Submitted to Biomedical Journals ICMJE criteria.

Acknowledgments: None

Authors' contributions: 
- Study concept and design: Duron Emmanuelle, Baudouin Edouard

- Acquisition of data: Kosowski Jill, Duron Emmanuelle

- Analysis and interpretation of data: Baudouin Edouard, Vidal Jean-Sébastien

- Drafting of the manuscript: Kosowski Jill, Baudouin Edouard

- Critical revision of the manuscript for important intellectual content: Mésinèle Léa, Pujol Tom, Brunetti Nicoletta, Colas Marion, Neiss Marie, Simon Pauline, Trivalle Christophe, Tiramine Soraya, Laraaj Fahd, Vetillard Anne-Laure, Houenou-Quenum Nadège, Souques Cécile, Verdier Sébastien, Houdre Julie, Sorrel-Dejerine Adrien, Sanchez-Tamayo Jorge, Vidal Jean-Sébastien, Collarino Rocco, Guichardon Magali, Jean-Emmanuel Kahn.

\section{Funding: This work was supported only by institutional sources.}

Availability of data and materials: The datasets used during the current study is available from the corresponding author on reasonable request

\section{References}

1. COVID-19 situation update worldwide, as of week 40, updated 14 October 2021 [Internet]. [cited 2021 Oct 18]. Available from: https://www.ecdc.europa.eu/en/geographical-distribution-2019-ncov-cases

2. SPF. COVID-19: point épidémiologique du 5 novembre 2020 [Internet]. [cited 2020 Dec 15]. Available from: /maladies-et-traumatismes/maladies-et-infections-respiratoires/infection-acoronavirus/documents/bulletin-national/covid-19-point-epidemiologique-du-5-novembre-2020

3. Wu Z, McGoogan JM. Characteristics of and Important Lessons From the Coronavirus Disease 2019 (COVID-19) Outbreak in China: Summary of a Report of 72314 Cases From the Chinese Center for Disease Control and Prevention. JAMA. 2020 Apr 7;323(13):1239.

4. Chen T, Wu D, Chen H, Yan W, Yang D, Chen G, et al. Clinical characteristics of 113 deceased patients with coronavirus disease 2019: retrospective study. BMJ. 2020 Mar 26;m1091.

5. Wang D, Hu B, Hu C, Zhu F, Liu X, Zhang J, et al. Clinical Characteristics of 138 Hospitalized Patients With 2019 Novel Coronavirus-Infected Pneumonia in Wuhan, China. JAMA. 2020 Mar 17;323(11):1061.

6. Zhou F, Yu T, Du R, Fan G, Liu Y, Liu Z, et al. Clinical course and risk factors for mortality of adult inpatients with COVID-19 in Wuhan, China: a retrospective cohort study. The Lancet. 2020 Mar;395(10229):1054-62. 
7. Richardson S, Hirsch JS, Narasimhan M, Crawford JM, McGinn T, Davidson KW, et al. Presenting Characteristics, Comorbidities, and Outcomes Among 5700 Patients Hospitalized With COVID-19 in the New York City Area. JAMA. 2020 May 26;323(20):2052.

8. Zerah L, Baudouin É, Pépin M, Mary M, Krypciak S, Bianco C, et al. Clinical Characteristics and Outcomes of 821 Older Patients With SARS-Cov-2 Infection Admitted to Acute Care Geriatric Wards. Newman AB, editor. J Gerontol Ser A. 2020 Aug 26;glaa210.

9. Katz S, Akpom CA. 12. Index of ADL: Med Care. 1976 May;14(Supplement):116-8.

10. Charlson M, Szatrowski TP, Peterson J, Gold J. Validation of a combined comorbidity index. J Clin Epidemiol. 1994 Nov 1;47(11):1245-51.

11. Pathophysiology, Transmission, Diagnosis, and Treatment of Coronavirus Disease 2019 (COVID19): A Review | Critical Care Medicine | JAMA | JAMA Network [Internet]. [cited 2020 Dec 15]. Available from: https://jamanetwork.com/journals/jama/fullarticle/2768391\%20//

12. Yale IMPACT Team, Lucas C, Wong P, Klein J, Castro TBR, Silva J, et al. Longitudinal analyses reveal immunological misfiring in severe COVID-19. Nature. 2020 Aug 20;584(7821):463-9.

13. Rodrigo-Muñoz JM, Sastre B, Cañas JA, Gil-Martínez M, Redondo N, Del Pozo V. Eosinophil Response Against Classical and Emerging Respiratory Viruses: COVID-19. J Investig Allergol Clin Immunol. 2020 Jun 16;0.

14. Gil H, Magy N, Mauny F, Dupond J-L. [Value of eosinopenia in inflammatory disorders: an "old" marker revisited]. Rev Med Interne. 2003 Jul;24(7):431-5.

15. Lavoignet C-E, Le Borgne P, Chabrier S, Bidoire J, Slimani H, Chevrolet-Lavoignet J, et al. White blood cell count and eosinopenia as valuable tools for the diagnosis of bacterial infections in the ED. Eur J Clin Microbiol Infect Dis Off Publ Eur Soc Clin Microbiol. 2019 Aug;38(8):1523-32.

16. Lavoignet CE, Le Borgne P, Slimani H, Forato M, Kam C, Kauffmann P, et al. [Relevance of eosinopenia as marker of sepsis in the Emergency Department]. Rev Med Interne. 2016 Nov;37(11):7304.

17. Abidi K, Belayachi J, Derras Y, Khayari ME, Dendane T, Madani N, et al. Eosinopenia, an early marker of increased mortality in critically ill medical patients. Intensive Care Med. 2011 Jul;37(7):113642.

18. Du Y, Tu L, Zhu P, Mu M, Wang R, Yang P, et al. Clinical Features of 85 Fatal Cases of COVID-19 from Wuhan. A Retrospective Observational Study. Am J Respir Crit Care Med. 2020 Jun 1;201(11):13729. 
19. Zhang J, Dong X, Cao Y, Yuan Y, Yang Y, Yan Y, et al. Clinical characteristics of 140 patients infected with SARS-CoV-2 in Wuhan, China. Allergy. 2020 Jul;75(7):1730-41.

20. Wang Z, Yang B, Li Q, Wen L, Zhang R. Clinical Features of 69 Cases With Coronavirus Disease 2019 in Wuhan, China. Clin Infect Dis. 2020 Jul 28;71(15):769-77.

21. Yun H, Sun Z, Wu J, Tang A, Hu M, Xiang Z. Laboratory data analysis of novel coronavirus (COVID19) screening in 2510 patients. Clin Chim Acta Int J Clin Chem. 2020 Aug;507:94-7.

22. Liu F, Xu A, Zhang Y, Xuan W, Yan T, Pan K, et al. Patients of COVID-19 may benefit from sustained Lopinavir-combined regimen and the increase of Eosinophil may predict the outcome of COVID-19 progression. Int J Infect Dis IJID Off Publ Int Soc Infect Dis. 2020 Jun;95:183-91.

23. Abidi K, Khoudri I, Belayachi J, Madani N, Zekraoui A, Zeggwagh AA, et al. Eosinopenia is a reliable marker of sepsis on admission to medical intensive care units. Crit Care Lond Engl. 2008;12(2):R59.

24. Shaaban H, Daniel S, Sison R, Slim J, Perez G. Eosinopenia: Is it a good marker of sepsis in comparison to procalcitonin and C-reactive protein levels for patients admitted to a critical care unit in an urban hospital? J Crit Care. 2010 Dec;25(4):570-5.

25. COVID-19 Clinical management: living guidance [Internet]. [cited 2021 Jul 23]. Available from: https://www.who.int/publications-detail-redirect/WHO-2019-nCoV-clinical-2021-1

26. Hemogram.pdf [Internet]. [cited 2021 Jul 23]. Available from: https://www.hassante.fr/upload/docs/application/pdf/Hemogram.pdf

27. Kellum JA, Lameire N. Diagnosis, evaluation, and management of acute kidney injury: a KDIGO summary (Part 1). Crit Care. 2013;17(1):204.

28. Seymour CW, Liu VX, Iwashyna TJ, Brunkhorst FM, Rea TD, Scherag A, et al. Assessment of Clinical Criteria for Sepsis: For the Third International Consensus Definitions for Sepsis and Septic Shock (Sepsis-3). JAMA. 2016 Feb 23;315(8):762.

29. Xie G, Ding F, Han L, Yin D, Lu H, Zhang M. The role of peripheral blood eosinophil counts in COVID-19 patients. Allergy [Internet]. [cited 2020 Dec 15];n/a(n/a). Available from: https://onlinelibrary.wiley.com/doi/abs/10.1111/all.14465

30. Tanni F, Akker E, Zaman MM, Figueroa N, Tharian B, Hupart KH. Eosinopenia and COVID-19. J Am Osteopath Assoc. 2020 Aug 1;120(8):504.

31. Azkur AK, Akdis M, Azkur D, Sokolowska M, Veen W van de, Brüggen M-C, et al. Immune response to SARS-CoV-2 and mechanisms of immunopathological changes in COVID-19. Allergy. 2020;75(7):1564-81. 
32. Le Borgne P, Abensur Vuillaume L, Alamé K, Lefebvre F, Chabrier S, Bérard L, et al. Do Blood Eosinophils Predict in-Hospital Mortality or Severity of Disease in SARS-CoV-2 Infection? A Retrospective Multicenter Study. Microorganisms. 2021 Feb 8;9(2).

33. Cazzaniga M, Fumagalli LAM, D'angelo L, Cerino M, Bonfanti G, Fumagalli RM, et al. Eosinopenia is a reliable marker of severe disease and unfavourable outcome in patients with COVID-19 pneumonia. Int J Clin Pract. 2021 Jan 26;e14047.

34. Ho KS, Howell D, Rogers L, Narasimhan B, Verma H, Steiger D. The relationship between asthma, eosinophilia, and outcomes in coronavirus disease 2019 infection. Ann Allergy Asthma Immunol Off Publ Am Coll Allergy Asthma Immunol. 2021 Feb 27;

35. Ferastraoaru D, Hudes G, Jerschow E, Jariwala S, Karagic M, de Vos G, et al. Eosinophilia in Asthma Patients Is Protective Against Severe COVID-19 Illness. J Allergy Clin Immunol Pract. 2021 Mar;9(3):1152-1162.e3.

36. Kovacs EJ, Boe DM, Boule LA, Curtis BJ. Inflammaging and the Lung. Clin Geriatr Med. 2017 Nov;33(4):459-71.

37. Jamilloux Y, Henry T, Belot A, Viel S, Fauter M, El Jammal T, et al. Should we stimulate or suppress immune responses in COVID-19? Cytokine and anti-cytokine interventions. Autoimmun Rev. 2020 Jul;19(7):102567.

38. Polverino F, Stern DA, Ruocco G, Balestro E, Bassetti M, Candelli M, et al. Comorbidities, Cardiovascular Therapies, and COVID-19 Mortality: A Nationwide, Italian Observational Study (ItaliCO). Front Cardiovasc Med [Internet]. 2020 [cited 2021 Jul 23];0. Available from: https://www.frontiersin.org/articles/10.3389/fcvm.2020.585866/full

39. Bass DA. Behavior of eosinophil leukocytes in acute inflammation. II. Eosinophil dynamics during acute inflammation. J Clin Invest. 1975 Oct 1;56(4):870-9.

40. European Society for Swallowing Disorders - European Union Geria | CIA [Internet]. [cited 2020 Dec 15]. Available from: https://www.dovepress.com/european-society-for-swallowing-disorders-ndasheuropean-union-geriatr-peer-reviewed-article-CIA

41. Barton LM, Duval EJ, Stroberg E, Ghosh S, Mukhopadhyay S. COVID-19 Autopsies, Oklahoma, USA. Am J Clin Pathol. 2020 May 5;153(6):725-33.

42. Lindsley AW, Schwartz JT, Rothenberg ME. Eosinophil responses during COVID-19 infections and coronavirus vaccination. J Allergy Clin Immunol. 2020;146(1):1-7.

43. Jesenak M, Banovcin P, Diamant Z. COVID-19, chronic inflammatory respiratory diseases and eosinophils-Observations from reported clinical case series. Allergy. 2020;75(7):1819-22. 
44. Gauckler P, Shin JI, Mayer G, Kronbichler A. Eosinophilia and Kidney Disease: More than Just an Incidental Finding? J Clin Med. 2018 Dec 8;7(12):529

\section{Figures}

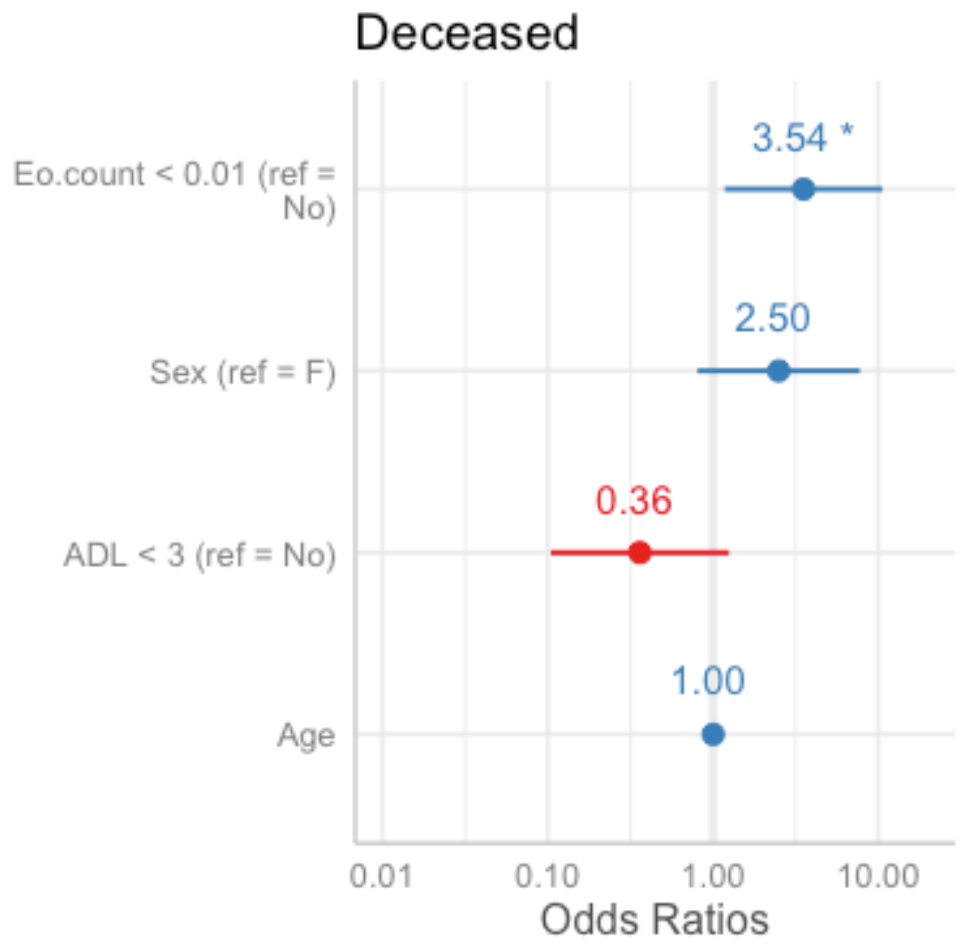

\section{Figure 1}

Multivariate analysis (deceased status adjusted on age, sex, eosinophil count and functional status), Forest Plot OR (95\% Cl): Age: 1.0 (0.92-1.10), Sex (ref = F): 2.50 (0.80-7.92), Eosinophil count: 3.54 (1.2311.4), $A D L<3($ ref = No): $0.36(0.09-1.17)$ 